treponemal antibody status was determined using the Architect ${ }^{\circledR}$ Syphilis TP CMIA (Abbott) as a screening test.

Results The TP-RT specificity was determined at $99.4 \%$ and the sensitivity at $97.0 \%$. For $12.2 \%$ of the positive sera the TP-RT demonstrated only borderline findings. From a subset of 135 positive samples the TP-RT showed reactivity after $15 \mathrm{~min}$ in 121 (89.7\%), $30 \mathrm{~min}$ in $129(95.6 \%), 60 \mathrm{~min}$ in $131(97.0 \%)$ and after $24 \mathrm{~h}$ in all of $135(100 \%)$. More than $70 \%$ of the positive findings were related to past treponemal infections. In two cases of primary syphilis a borderline TP-RT result only occurred after 45 to $60 \mathrm{~min}$.

Discussion The TP-RT is not able to differentiate active from past treponemal infections. Any reactive TP-RT finding has to be further tested by conventional syphilis tests. Negative TP-RT results after a reading time from 15 to 30 minutes does not exclude the presence of highly infectious early stages of syphilis. A minimum reading time of 1-2 hours is recommended. The high number of borderline findings even in active syphilis cases poses the risk of incorrect assessment of the test results. The routine use of rapid tests is not recommended for syphilis diagnosis in Germany.

\section{P2.069* FACTORS ASSOCIATED WITH BIOLOGIC FALSE POSITIVE RAPID PLASMA REAGIN (RPR) SEROLOGIES IN HIV-1- INFECTED PERSONS}

doi:10.1136/sextrans-2013-051184.0334

I Oboho, K Gebo, R Moore, K G Ghanem. Johns Hopkins University, Baltimore, MD, United States

Objectives The prevalence of biologic false-positive (BFP) nontreponemal tests ranges from $4-15 \%$ among HIV-infected persons. Abnormalities in B cell function are hypothesised to increase the probability of BFP in this population. Our aim was to determine the impact of combination antiretroviral therapy (cART) and the degree of immunosuppression on BFP Rapid Plasma Reagin (RPR) tests among HIV-infected persons.

Methods We conducted a retrospective study of 711 HIV-infected patients enrolled in the Johns Hopkins HIV Clinical Cohort. BFP RPR was defined as a reactive RPR and a non-reactive FTA-ABS. We conducted two analyses: (1) A cross-sectional analysis in which patients with BFP tests were compared to two control groups: HIVinfected patients (i) with syphilis and (ii) without syphilis. (2) A longitudinal analysis to determine the factors associated with BFP persistence over time. A persistent BFP test was defined as a BFP test at all visits in patients who had more than one visit with a documented RPR test. We used logistic regression and Generalized Estimating Equations for the analyses.

Results 96 participants (13.5\%) had BFP tests and 273 (48.1\%) had syphilis. Twenty-two of $96(23 \%)$ had persistent BFP tests. cART use was associated with decreased odds of BFP tests compared to persons with syphilis [adjusted odds ratio (aOR) 0.31, 95\% CI: 0.15-0.63] and without syphilis [aOR $0.42(0.22-0.81)$ ]. cART use was also associated with decreased odds of BFP persistence over time [OR $0.07(0.01-0.33)]$. Neither CD4 count nor HIV RNA was associated with BFP test results. Lower RPR titers, injection drug use and Hepatitis B were associated with increased odds of BFP.

Conclusions The use of cART appears to significantly decrease the odds of BFP RPR tests, independent of CD4 T-cell response. This may be the result of cART's effects on B-cell functions.

\section{P2.070 NO MISCLASSIFICATION OF SYPHILIS CASES USING A REVERSE SEOUENCE ALGORITHM IN REACTIVE ENZYME IMMUNOASSAY AND REACTIVE RPR SAMPLES WHEN RPR TITER ABOVE 1:2}

doi:10.1136/sextrans-2013-051184.0335
C Fortin, ${ }^{2} \mathrm{~A} C$ Labbé, ${ }^{3} \mathrm{~L}$ Côté, ${ }^{4} \mathrm{~J}$ Fafard, ${ }^{5} \mathrm{~L}$ Delorme, ${ }^{6} \mathrm{~A}$ Trudelle, ${ }^{7} \mathrm{C}$ Tremblay, ${ }^{7} \mathrm{~B}$ Serhir 'CHUM: Hôpital Notre-Dame, Montreal, OC, Canada; ${ }^{2}$ Hopital Maisonneuve-Rosement, Montreal, OC, Canada; ${ }^{3} \mathrm{CHUO}$ : Centre Hospitalier de I'Université Laval, Quebec City, OC, Canada; ${ }^{4}$ Centre Hospitalier Pierre Le-Gardeur, Terrebonne, OC, Canada; ${ }^{5} \mathrm{Hôpital}$

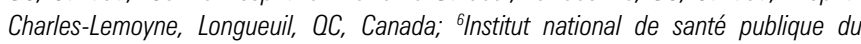
Québec, Montreal, OC, Canada; 'Laboratoire de santé publique du Québec, Montreal, OC, Canada

Background Recent recommendations propose that serum samples reactive by both syphilis enzyme immunoassay (EIA) or chemoluminescent immonoassay (CLIA) and RPR may not need treponemal confirmatory testing. There is uncertainty regarding the confirmation rate of EIA/CLIA reactive and low titer RPR samples.

Methods Reactive samples for EIA/CLIA and low titer RPR from five Quebec diagnostic laboratories between December $14^{\text {th }}$ 2011 and December $3^{\text {rd }} 2012$ were prospectively tested with TPPA and, if negative or inconclusive, with a line immunoassay (LIA). Syphilis infection confirmation was defined by a reactive TPPA or LIA.

Results Samples reactive for EIA/CLIA and RPR with titers ranging from 1:1 to 1:8 were submitted for confirmatory testing $(\mathrm{N}=345)$. Of these, 335 (97.1\%) were confirmed and 2.9\% (95\% CI $1.4-5.3 \%$ ) were misclassified as syphilis cases. When stratifying by RPR titer, unconfirmed samples (misclassified cases) were found only in samples with RPR titer of 1:1 and 1:2. Samples with titers above 1:2 were classified as true syphilis cases. Proportion of confirmed cases increased with RPR titer $(p=0.01)$.

Abstract P2.070 Table 1 Confirmatory testing results in samples according to RPR titer

\begin{tabular}{llllll}
\hline & N & TPPA reactive & LIA reactive & Unconfirmed & Confirmed \\
\hline All titers & 345 & $326 / 345$ & $9 / 19$ & $10(2.9 \%)$ & $335(97.1 \%)$ \\
RPR 1:1 & 112 & $99 / 112$ & $5 / 13$ & $8(7.1 \%)$ & $104(92.9 \%)$ \\
RPR 1:2 & 104 & $100 / 104$ & $2 / 4$ & $2(1.9 \%)$ & $102(98.1 \%)$ \\
RPR 1:4 & 74 & $73 / 74$ & $1 / 1$ & 0 & $74(100 \%)$ \\
RPR 1:8 & 55 & $54 / 55$ & $1 / 1$ & 0 & $55(100 \%)$ \\
\hline
\end{tabular}

Conclusions In our setting, only patients with serum RPR titers ranging from 1:1 to $1: 2$ would have been misclassified as syphilis cases had a confirmatory test not been conducted. A safe and costeffective approach, for EIA/CLIA reactive and RPR reactive samples management in a reverse sequence algorithm may be to submit only samples with low RPR titers $(\leq 1: 2)$ instead of all EIA/CLIA and $\mathrm{RPR}$ reactive samples for confirmation.

\section{P2.071 SUPERIOR DETECTION OF SYPHILIS WITH THE RAPID TEST DETERMINE ${ }^{\circledR}$ COMPARED TO COMBINED CARDIOLIPIN AND TREPONEMAL SPECIFIC TESTS}

doi:10.1136/sextrans-2013-051184.0336

'S G Chapman, 'P Fernández Cañadas, 2J Martín Lluch. ' Centro Médico Open House, Madrid, Spain; '2Laboratorios Ruiz Falcó, Madrid, Spain

Objectives To quantify detection of syphilis with the DETERMINE $^{\circledR}$ Syphilis test versus single or combined treponemal/cardiolipin tests in a Primary Care setting.

Methods The 107 positive DETERMINE ${ }^{\circledR}$ syphilis results obtained in 1898 samples were compared to combined RPR/ VDRL and FTA results. True positives were defined as either serological confirmation by either cardiolipin and/or specific treponemal positive results at diagnosis or at 1 week follow-up, or signs and symptoms classically compatible with syphilis which resolved completely and rapidly on administration of single dose benzathine penicillin G. All positive cases showed a sustained positive 